\title{
A Comprehensive Healthcare System to Detect Depression
}

\author{
M. Poonkodi*, Ananya Srinivasan, Bhavana Tumma and Srividya Ramaswamy \\ SRM University, 100 Feet Road, Vadapalani, Chennai -600026, Tamil Nadu, India; poonkodi1679@gmail.com, \\ srinivasan.ananya95@gmail.com, bhavana.tumma@gmail.com, srividya.ramaswamy23@gmail.com,
}

\begin{abstract}
Objectives: To develop a home-based healthcare unit that uses smart phones to monitor depression statistics. Methods: To tackle depression, we propose a mechanism which systematically monitors the user's behavior on a daily basis. We make use of a Smartphone to obtain GPS and phone usage data from the user and employ a clinically approved survey (PHQ-9) to monitor their mental state. The result obtained from computation determines the level of depression of the given user which is then sent to the consulting physician. Findings: Depression is one of the major psychological issues present in today's world, which often goes unnoticed because of its subtle symptoms and social stigma. Also, that people are too engrossed within their daily duties does not help them realize the problem even when depression related symptoms prevail. A study based on the World Health Organization's World Mental Health Survey Initiative has said that India has the highest rate of major depression in the world. An untimely detection of depression related symptoms may prove to be detrimental to several vital parameters of the body. By the proposed implementation, the physician can conveniently monitor the user's health status periodically without spending much time explicitly being a part of the process. The application of this healthcare system is to detect the onset of depression and prevent drastic results such a suicide and help the patient be in contact with their physician at all times. Improvements: Future improvements include, making the whole process less invasive by implementing Medical Imaging (Brain Scans) and incorporating IoT to increase parameters in the decision making matrix of depression detection.
\end{abstract}

Keywords: Cluster, DBSCAN, PHQ-9, GPS, Phone Usage

\section{Introduction}

Depression is a state of low mood and aversion to activity that can affect a person's thoughts, behavior, feelings and sense of well-being. People with depressed mood can feel sad, anxious, empty, hopeless, helpless, worthless, guilty, irritable, ashamed or restless. It is also the major cause of insomnia, excessive sleeping, fatigue, aches, digestive problems etc. Out of every 10 Indian professionals, 4 suffer from general anxiety disorder or depression. In the list of the top diseases that affect corporate executives, depression (42\%) ranks at the top, followed by obesity (23\%), high blood pressure (9\%) and diabetes (8\%). Over half (55\% to be precise) are under the age of 30 and a quarter are between 30 and 40 years ${ }^{1}$. A study based on the World Health Organization's World Mental Health
Survey Initiative has said that India has the highest rate of major depression in the world ${ }^{2-4}$. Depression is most commonly treated using psychotherapy and antidepressants. Antidepressants cannot cure depression, but only make it easier for you to deal with it ${ }^{5,6}$. However, antidepressants given at the wrong time can lead to adverse effects that go unnoticed ${ }^{\mathrm{T}}$. Antidepressants are not always the correct solution for curing depression. It is important to determine the level of depression a person is suffering from in order to provide them with the appropriate treatment. Thus to determine this, we aim at developing a home-based healthcare unit that uses Smartphone to monitor statistics and hence, detecting depression at the right time with the right parameters.

Sensors available in Smartphone give an accurate account of the user's behavior. Global Positioning System or

${ }^{*}$ Author for correspondence 
GPS sensor available on the phone tracks the movements of the user to determine his home, work and other frequently visited places ${ }^{8}$. We also extract other information like phone usage frequency, phone usage duration, apps used and first and last usage times to determine the behavior pattern of the user. A periodic update of these data to the server allows the system to compute the appropriate results and display it at the physician's end. The user is also required to fill in a survey that is clinically approved. This survey determines the mental status of the patient. The Phone Usage statistics is also represented graphically for easier understanding of the data. The computed value from the phone usage along with the answers provided by the user is monitored by the consulting physician periodically, hence giving the automated system a human eye for monitoring.

The phases involved in the process are:

1. Data Pre-processing

2. Feature Extraction

3. Estimating Level of Depression

The following are the assumptions made for the working of the proposal:

1. System is targeted towards detection of onset of depression.

2. Target patients are of the ages between 18 and 33 .

3. Night shift workers are not considered.

4. Made for a developing country with the infrastructure supporting Wi-Fi connectivity on the go.

5. Used to detect the depression level based on Smartphone Usage of an individual.

\section{Materials and Methods}

\subsection{Mind At Ease-android Application}

Mind At Ease is an open-source Android Application. It is developed with an aim to make user data collection easier and efficient. Mind At Ease employs a store and forward architecture which collects the phone usage data and stores it in the local device memory and then forwards it to the server when internet becomes available. This helps us in keeping track of the phone usage data even when the user does not find intermittent access to network facility and thus preventing data loss.

Mind At Ease keeps the identity of the user anonymous before storage and transmission. It makes use of the AES encryption algorithm to achieve the same. After encryption the data is stored in the user's device and deleted once it is uploaded to the server. At the physician's end the decryption is performed before displaying the information.

Overall, it collects user's location context using GPS, various application usage and other phone usage statistics. The GPS is recorded every 5 minutes and other information is collected through screen lock events and phone usage data per day ${ }^{8}$.

The interface of the app is shown in Figures 1-4 represents PHQ-9 survey.

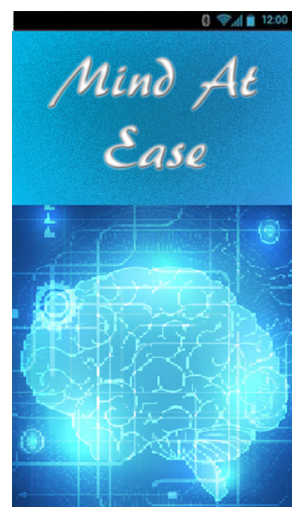

Figure 1. Start. Screen

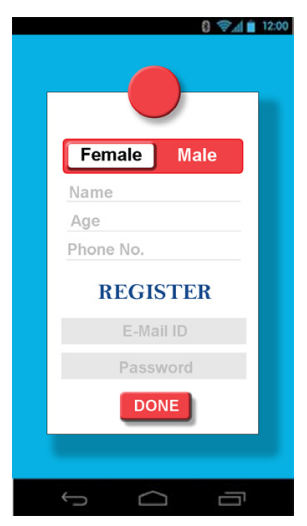

Figure 2. Registration screen.

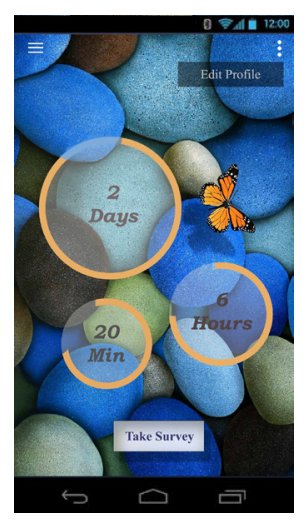

Figure 3. Time remaining for the survey. 


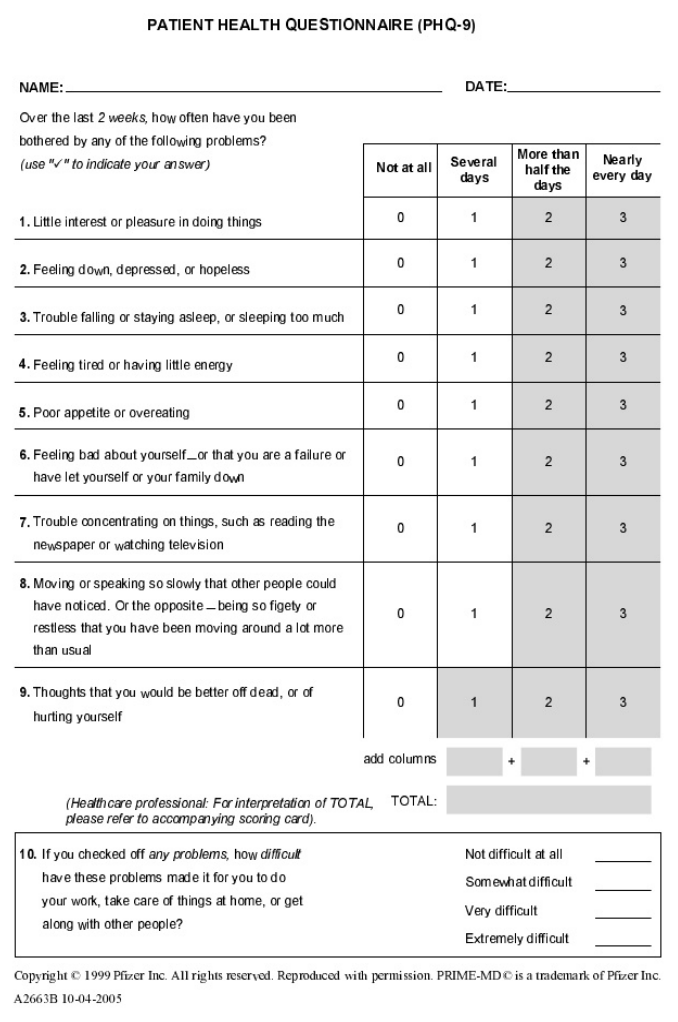

Figure 4. PHQ- 9 Survey.

\subsection{Data Preprocessing}

In this phase we look to collect data from the user's Smartphone. We divide this approach into two parts; Manual and automatic. In the manual approach we collect the data in the form of a self-reported survey which is a combination of PHQ-9 Survey and a set of questions recommended by psychiatrists. The PHQ-9 Survey is a set of ten questions that are clinically approved and is primarily used to detect depression symptoms in a user. The other set of questions are based on the user's lifestyle such as academic or work related achievements, goals, alcohol consumption etcetera. Survey is self-reported weekly by the user.

The automatic approach uses the phone usage as the foundation. Phone usage includes the following features:

1. GPS data

2. Phone Usage Frequency

3. Phone Usage Data

4. Apps used by the patient

5. First and Last Phone Usage times

Unlike the Survey data, these usage statistics are collected daily. Once collected the data is uploaded periodically to the server. Furthermore, as it involves confidential medical information, the data is encrypted and sent.

\subsection{Feature Extraction}

The next step in the procedure is to extract various features from the information derived using the Smartphone sensors ${ }^{9}$. Location Data extracted is used to identify the various places visited by the user in that week. This is done by identifying the different location clusters formed from the latitude and longitude values of the places visited in the week. This is achieved using the clustering algorithm through which the GPS information collected will be segregated into separate clusters and its centroid point will be plotted on to a map. These cluster points are of high importance when extracting features from the same. For this process we have used DBSCAN (Density-based spatial clustering of applications with noise). When provided a set of points in space, it groups them on the basis of how closely they are packed together and marks as outliers, points that lie alone in low-density regions ${ }^{10}$.

The features extracted from the Phone statistics are

1. Location Variance

2. Number of Clusters

3. Entropy

4. Normalized Entropy

5. Home Stay

6. Phone Usage Frequency

7. Phone Usage Duration

\subsubsection{Location Variance}

We measure the variability in a participant's GPS location. To calculate this, we use the location data of stationary states. It is computed as the logarithm of the sum of the statistical variances of the latitude and the longitude components of the location data:

$$
\text { Location Variance }=\log _{10}\left(\sigma_{\text {lat }}{ }^{2}+\sigma_{\text {long }}^{2}\right)
$$

The logarithm has been applied to compensate for the skewness in the distribution of location variance.

\subsubsection{Number of Clusters}

It is the number of clusters found by the DBSCAN algorithm in the preprocessing stage.

\subsubsection{Entropy}

Entropy is the measure of the variability of the time a participant spends at the location clusters. It is given as: 


$$
\text { Entropy }=-\sum_{\mathrm{i}} \mathrm{P}_{\mathrm{i}}\left(\log _{10} \mathrm{P}_{\mathrm{i}}\right)
$$

where,

$\mathrm{i}=1,2 \ldots \mathrm{N}$ represents a location cluster

$\mathrm{N}=$ denotes the total number of location clusters

$\mathrm{P}_{\mathrm{i}=}$ Percentage of time the participant spent at the location cluster ' $\mathrm{i}$ '

High entropy indicates that the participant spent time more uniformly across different location clusters and lower entropy indicates greater inequality in the time spent across the clusters.

\subsubsection{Normalized Entropy}

We divide the entropy by its maximum value, which is the logarithm of the total number of clusters.

$$
\text { Normalized Entropy }=\text { Entropy } / \log _{10} \mathrm{~N}
$$

Normalized entropy is invariant to the number of clusters and thus depends solely on the distribution of the visited location clusters. The value of normalized entropy ranges from $0-1$, where 0 indicates that all location data points belong to the same cluster and 1 implies that they are uniformly distributed across all the clusters.

\subsubsection{Home Stay}

We Measure the percentage of time a participant spends at home relative to other location clusters. For this we find the participant's home cluster. The home cluster is identified based on two heuristics:

1. The home cluster is among the first to the third most visited clusters

2. The home cluster is the cluster most visited during the time period between 12 a.m. and 6 a.m. (participants having night shift are not taken into account)

\subsubsection{Phone Usage Frequency}

It is on average, how many times during a day a participant interacts with their phone.

\subsubsection{Phone Usage Duration}

It is on average, the total time in seconds that a participant spends each day interacting with their phone.

\subsection{Correlating Features with Level of Depression}

Once the data is collected, we aim to correlate each feature with the PHQ-9 score of the user. The PHQ-9 is used as a provisional diagnosis method based on the user's self-reported survey answers which return a scaled score. The score defines the level of depression of the user. If the user is classified as not depressed his PHQ- $9<5$, else he is classified with depressive symptoms (PHQ-9 $\geq 5$ ). Finally, the correlation is applied to determine how strongly each feature maps to the PHQ-9 scores $\frac{11,12}{}$.

\section{Results and Discussion}

Once the listed features are extracted and processed, we estimate the level of depression. This is done by standard feed-forward Back Propagation Neural Network. The input layer contains 21 Input Neurons and the output layer contains 1 output neuron. The numbers of hidden layer neurons are determined through trial and error. The network undergoes a training process where the output is compared to a desired output; the computed error is then used to correct the weights. The weights are set to the following inputs:

1. A set of ten questions suggested by the psychiatrist.

2. Location Variance

3. Entropy

4. Normalized Entropy.

5. Home Stay

6. Phone Usage Frequency

7. Phone Usage Duration

8. Sleep Duration

9. Gender

10. Age

11. Type of App Used

12. PHQ-9 score

The activation function is the sigmoid function. It is given as:

$$
\mathrm{S}(\mathrm{t})=1 / 1+\mathrm{e}^{-\mathrm{t}}
$$

Its range contains values between zero and one. And thus, we normalize all inputs to the same range. A threshold is chosen in such a way that the output neuron fires for the given input neurons. The output value is mapped and displayed on the web application to the physician $\frac{13,14}{2}$.

\subsection{Physician's Portal}

The web application forms the physician's portal wherein he can keep a periodic track of the user's movement and phone usage. Apart from these, the physician can also view the answers given to the survey questions by the user 
along with the scaled score ${ }^{15}$. The web application provides a detailed and structured layout of the information. It also allows the physician to have a list of all his patients and a comprehensive perspective of each patient's data. In addition, the physician can also view and analyze weekly data in a systematic manner. In case of any anomalies, the physician can e-mail the user to meet him for a check-up.

The graphical representations are shown in Figures 5 and 6.

\subsection{Future Enhancements}

The scope of the above explained concept is beyond this project. Incorporated with Internet of Things $\frac{16}{6}$ and machine learning algorithms, implemented from the physician side will make this real time project helpful to a wide range of public struggling with the starting stages of depression. The future enhancements include

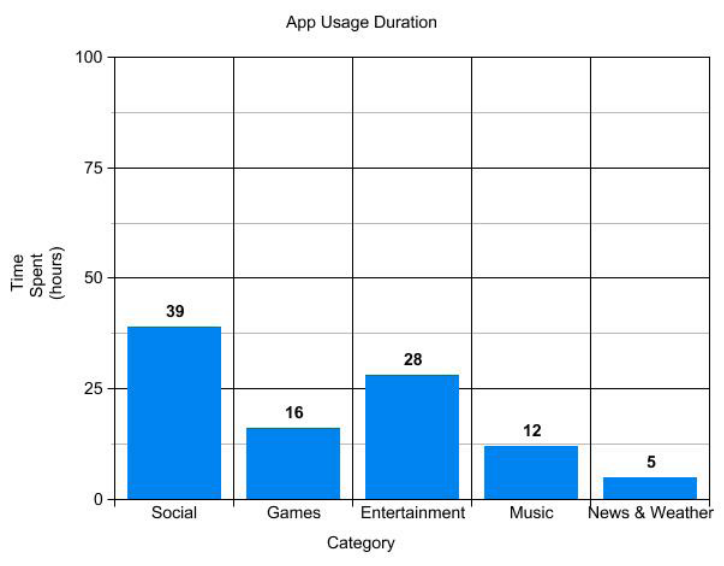

Figure 5. App Usage Duration.

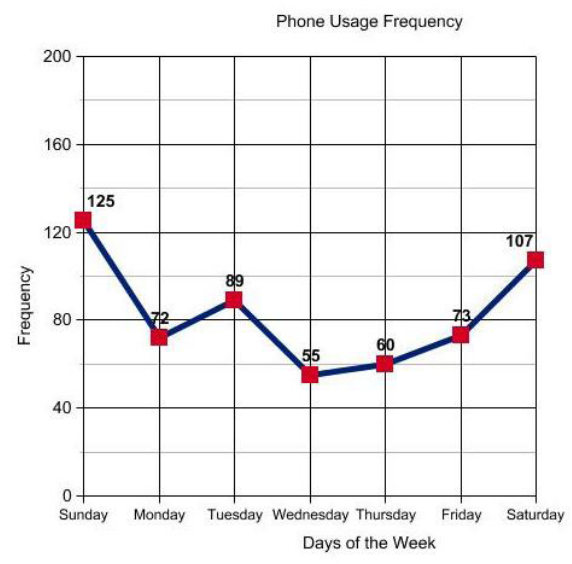

Figure 6. Phone Usage Frequency.
1. Embedding IoT in the household furniture such as the bed, the lamp and the windows to monitor sleep time and active time.

2. Interconnection of all the appliances such as television, media players and computers to sort out the mood and activities of the user.

3. Transmission of all this data to the physician's side and timely suggestions on health care improvement.

On reaching a threshold limit, when the depression level is increasing, the physician will suggest the patient to take a brain scan. A machine learning algorithm will be employed on the physician's end to classify the severity or depression and also detect other neurological problems. This ultimately results in a $100 \%$ accurate and non invasive method to detect chronic depression and start appropriate treatment immediately.

\section{Conclusion}

Depression is one of the most documented conditions in the world but one of the least discussed topic. It is important to understand that it is not something to be ignored. Only $20 \%$ of the World's depressed population seeks help for treatment due to the stigma and social opinions attached to it. This paper is the first step towards overcoming this situation. An automated system that keeps track of the user behavior to compute the depression level without much of an interaction makes the functioning of the physician easier as compared to a session of therapy or a dose of antidepressants. Also, the periodic monitoring of the extracted data allows the physician to suggest the appropriate remedy required instead of providing treatment that might have catastrophic effects on the patients in the long run.

\section{Acknowledgement}

We are grateful to our professor, Mrs. M. Poonkodi, for having assisted and mentored us so diligently in the process of preparing our project. Without her persistent support and co-operation, we couldn't have accomplished our ideas.

\section{References}

1. Yeun EJ, Jeon M. Level of depression and anxiety among undergraduate students. Indian Journal of Science and Technology. 2015 Dec; 8(35):1-5. 
2. We need to talk about depression: Darryl Neher at TEDxBloomington, video, Channel TEDx Talks [Internet]. 2013 May 4. [Cited 2015 Nov 01]. Available from: https:// www.youtube.com/watch?v=NvbWKwxA6YY.

3. Doryab A, Min JK, Wiese J, Zimmerman J, Hong J. Detection of behavior change in people with depression, Modern Artificial Intelligence for Health Analytics AAAI; 2014. p. 12-16.

4. Saeb S, Zhang M, Karr CJ, Schueller SM, Corden ME, Kording K, Mohr DC. Mobile phone sensor correlates of depressive symptom severity in daily-life behavior: An exploratory study. Journal of Medical Internet Research. 2015; 17(7):175.

5. Burns M, Begale M, Duffecy J, Gergle D, Karr C, Giangrande E, Mohr D. Harnessing context sensing to develop a mobile intervention for depression. Journal of Medical Internet Research. 2011; 13(3).

6. Why We Need to Talk about Depression | Kevin Breel | TED Talks, video, Channel Ted [Internet]. 2013 Sep 27. [Cited 2015 Dec 01]. Available from: https://www.youtube. com/watch?v=-Qe8cR4J110.

7. What Causes Depression.mov, video, Channel Depression Outreach's channel [Internet]. 2012 Mar 3. [Cited 2015 Dec 02]. Available from: https://www.youtube.com/ watch? $\mathrm{v}=\mathrm{InNhDfDfl5c}$.

8. Skubic M, Guevara RD, Rantz M. Automated health alerts using in-home sensor data for embedded health assessment. IEEE Journal of Transitional Engineering in Health and Medicine. 2015; 3:1-11.
9. Gao Y, Li H, XhuT. Predicting subjective well-being by smartphone usage behaviors. Proceedings of the International Conference on Health Informatics, ESEO, Angers, Loire Valley, France; 2013. p. 317-22.

10. Chittaranjan G, Blom J, Perez DG. Mining large-scale smartphone data for personality studies. International Symposium on Wearable Computers, San Francisco, California; 2011 Jun.

11. Grunerbl A, Muaremi A, Osmani V, Bahle G, Ohler S, Troster G, Mayora O, Haring C, Lukowicz P. Smartphone based recognition of states and state changes in bipolar disorder patients. IEEE Journal of Biomedical and Health Informatics. 2014; 19(1):140-8

12. Sowjanya AM, Shashi M. Cluster Feature-based Incremental Clustering Approach (CFICA) For numerical data. International Journal of Computer Science and Network Security. 2010; 10(9):73-9.

13. Hsu CC, Huang T. Incremental clustering of mixed data based on distance hierarchy. Expert Systems with Applications. 2008; 35(3):1177-85.

14. Halfin A. Depression: the benefits of early and appropriate treatment. The American Journal of Managed Care. 2007; 13(4):92-7.

15. Phillips JG, Butt S, Blaszczynski A. Personality and selfreported use of mobile phones for games. Cyberpsychology and Behavior. 2006; 9(6):753-8.

16. An YJ, Kim DH. Sensor web based on multiple interface for public environmental surveillance. Indian Journal of Science and Technology. 2015 Aug; 8(18):1-3. 